\title{
Kommunikation - Verständigung und Sprache in der Evaluation. Ein persönlicher Bericht zur 23. Jahrestagung der DeGEval, 17.-18.09.2020
}

\section{Evelyn Funk ${ }^{1}$}

Einmal im Jahr haben wir im deutschsprachigen Raum die Möglichkeit, uns über unsere persönlichen Netzwerke hinaus intensiv über das Thema Evaluation auszutauschen und so unseren Horizont zu erweitern: Mit Menschen aus anderen Politikfeldern, in anderen Positionen; mit Menschen, die andere Erfahrungen mitbringen und eine andere Sicht auf die Dinge haben. Dieser Austausch ist nicht nur inspirierend, sondern auch notwendig für die professionelle Weiterentwicklung von uns allen und von unserem Berufsfeld insgesamt.

In 2020 war aufgrund der COVID-19-Pandemie alles anders, so auch die die Jahrestagung der DeGEval. Bereits Anfang Mai war klar, dass ein persönliches $\mathrm{Zu}$ sammenkommen nicht möglich sein würde, und so wurde die Tagung zum ersten Mal in der Geschichte der DeGEval als reine Online-Tagung durchgeführt - mit sämtlichen Vor- und Nachteilen, die wir alle in diesem Jahr der Pandemie so intensiv kennengelernt haben.

Das Thema der Jahrestagung „Kommunikation - Verständigung und Sprache in der Evaluation" bekam durch die besonderen Umstände also noch eine zusätzliche Ebene: Die Teilnehmerinnen und Teilnehmer der Tagung haben sich nicht nur mit der Kommunikation innerhalb von Evaluationen und der Kommunikation über Evaluation in der breiteren Öffentlichkeit beschäftigt, sondern zwangsläufig auch mit der besonderen Kommunikationssituation der Tagung selbst.

Der eigentliche Ablauf der Tagung folgte dem bekannten Schema der letzten Jahre - nur eben virtuell. Die Weiterbildungsseminare, die sich als ergänzendes Angebot im Rahmen der Jahrestagung etabliert haben, fanden bereits in der Vorwoche statt. Karin Grasenick gab in ihrem Workshop Einblicke und Anregungen dazu, wie „Gender als Evaluierungskompetenz" konkret in der Evaluation umgesetzt werden kann. Stefan Rädiker führte in die computergestützte Analyse von qualitativen Daten mit der Software MAXQDA ein. Wie gewohnt begann der erste Tagungstag

1 PME-Campus, Frechen 
dann mit dem Treffen des Nachwuchsnetzwerks und den Arbeitskreistreffen. ${ }^{2}$ In diesen recht kurzen, intensiven Treffen haben die Teilnehmenden wie gewohnt aktuelle Entwicklungen in ihrem jeweiligen Arbeitsfeld thematisiert. Zur Illustration sei hier das Treffen des AK Entwicklungszusammenarbeit und Humanitäre Hilfe genannt: Hier gab es neben den „Neuigkeiten aus den Institutionen“ einen Input zur Reform der für die Entwicklungszusammenarbeit extrem wichtigen Evaluationskriterien des OECD-DAC und zum Umgang des BMZ damit; außerdem stellte sich die 2020 neu konstituierte Arbeitsgruppe „Remote Evaluation“ vor. Dieser kurze Ausschnitt zeigt: Die AK-Treffen sind eine gute Gelegenheit, sich einen Überblick über relevante Vorgänge und auch Austauschmöglichkeiten rund um das jeweilige Thema $\mathrm{zu}$ verschaffen. Und natürlich sind sie insofern wichtig, als dass sie uns in Erinnerung rufen, dass wir als AK-Mitglieder aktiv an der Gestaltung verschiedener spezieller Themenfelder im Bereich der Evaluation mitwirken dürfen.

Wie gewohnt kamen nach den Sitzungen der Arbeitskreise alle Teilnehmenden zur Tagungseröffnung zusammen. Jan Hense begrüßte alle Anwesenden im Namen der DeGEval, doch an dieser Stelle war ein Nachteil des virtuellen Formats ganz besonders spürbar: die Tagungsbesucherinnen und -besucher blieben unsichtbar. Für die Konzentration, aber auch für das Zusammengehörigkeitsgefühl einer Gruppe, macht es einen großen Unterschied, ob man sich in einem Saal versammelt und gemeinsam in ein Tagungsthema einsteigt, oder ob man jeweils alleine vor dem Bildschirm sitzt.

Nach der offiziellen Begrüßung ging das Wort dann an Monika Taddicken, Professorin für Kommunikationswissenschaft an der TU Braunschweig. Sie lud alle Anwesenden ein, mit ihrer Keynote „Eine Frage der Erwartungen? Eine kommunikationswissenschaftliche Perspektive auf Qualitätsbewertungen“ tiefer in das Tagungsthema einzutauchen. Unter anderem sprach sie über die „Lasswell-Formel“, die wesentliche Aspekte von Kommunikation in einer Frage zusammenfasst: WER sagt WAS über WELCHEN KANAL zu WEM mit WELCHER WIRKUNG?

Ich möchte diese Formel im Folgenden als Strukturierungshilfe nutzen, um ein paar Aspekte rund um das Thema Kommunikation aufzugreifen, die im Rahmen der Jahrestagung diskutiert wurden.

\section{WER?}

In fast allen Sessions haben die Teilnehmenden sich damit auseinandergesetzt, welche Rolle sie selbst in der Kommunikation spielen und welche Verantwortlichkeiten damit einhergehen. Und auch wenn in den meisten Evaluationssituationen alle Beteiligten eine Mitverantwortung für gelungene Kommunikation haben - die Evaluierenden sind meist ganz besonders in der Pflicht. In einer Session des AK Methoden, moderiert von Jan Tobias Polak, stellten Susanne Mäder, Anke Köhler, Myriam Karlinger und Karl Mühlbachler mit der Gruppendiskussion, dem Interview und der

2 Die Mitgliederversammlung, die üblicherweise am Vorabend der Jahrestagung stattfindet, wurde 2020 aufgrund der besonderen Umstände ausgelagert und fand einige Wochen später im November 2020, ebenfalls virtuell, statt. 
Peer-Befragung ganz konkrete Methoden mit hohem kommunikativem Potenzial vor, die Evaluierende nutzen können, um (per guter Kommunikation noch während des laufenden Prozesses) die Nützlichkeit von Evaluation zu erhöhen. Mit der Kommunikation von und dem Lernen aus Evaluationsergebnissen setzten sich unter anderem Franziska Pfitzner-Eden gemeinsam mit Gert Lang, Birgit Teufer, Petra Wagner und Marija Peric auseinander. Sie diskutierten beispielsweise, wie Evaluierende eine zielgruppenadäquate und umsetzungsorientierte Aufbereitung von Evaluationsergebnissen erreichen können und welche Faktoren Fördernde und Projektdurchführende dahin gehend unterstützen können, dass sie den Transfer in die Praxis gelungen gestalten.

\section{WAS (und WIE)?}

Das „was“ und „wie“ von Kommunikation rund um Evaluation wurde auf der Jahrestagung auf vielen verschiedenen Ebenen bearbeitet. In der 2. Keynote am Freitagmorgen mit dem Titel „Plädoyer für eine konsistente Fachsprache der Evaluation“ referierte Wolfgang Beywl, Seniorprofessor an der Pädagogischen Fachhochschule Nordwestschweiz, über unsere Fachsprache. Er stellte heraus, dass eine konsistente und reflektierte Fachterminologie nicht nur für unseren fachlichen Austausch untereinander eine wichtige Voraussetzung ist. Sie bietet darüber hinaus auch eine Basis für die Kommunikation über Evaluation mit Menschen außerhalb des Fachbereichs, zwingt uns zu mehr Genauigkeit und damit auch zu mehr Fairness. Wolfgang Beywls Keynote endete folgerichtig mit seinem Plädoyer dafür, dass wir die Evaluationsfachsprache weiter systematisieren und normieren. Als Plattform für diese gemeinschaftliche Anstrengung verwies er auf das deutschsprachige Glossar der Evaluation, das ständig nach dem Wiki-Prinzip demokratisch und partizipativ weiterentwickelt wird (https://eval-wiki.org/glossar/Eval-wiki.org).

Auch die 1. Keynote von Monika Taddicken befasste sich mit unserer Fachsprache - ihr Fokus lag jedoch auf der Kommunikation nach außen. Für diese spezielle Situation, die sich auch unter dem Begriff „Wissenschaftskommunikation“ subsummieren lässt, stellte sie eine Reihe von empirisch hergeleiteten Qualitätskriterien vor (Wicke/Taddicken 2020; Taddicken/Wicke/Willems 2020). Demnach wird Wissenschaftskommunikation als qualitativ hochwertig empfunden, wenn komplexe Themen verständlich dargestellt sind, wenn Prozesse und Methoden erklärt werden, wenn Unsicherheiten und Widersprüche von wissenschaftlichen Ergebnissen thematisiert werden, wenn die Vielfalt von Perspektiven dargestellt wird, wenn die Alltagsrelevanz wissenschaftlicher Fragen hervorgehoben wird und wenn auch Neuigkeits- und Unterhaltungswert berücksichtigt werden.

Einige dieser Aspekte spiegeln sich in den Evaluationsstandards der DeGEval, aber sie gehen doch weit darüber hinaus. An dieser Stelle zeigte sich für mich erneut, wie wertvoll der Blick über den Tellerrand der eigenen Fachdisziplin hinaus sein kann.

Ein paar konkretere Ideen dazu, wie gelungene Kommunikation in der Evaluation aussieht, diskutierte auch Georg Spiel in einer Session gemeinsam mit Ludwig 
Grillich, Elitza Uzunova, Hannah Pütz und Jan Karsten Gierse. Hier ging es um drei ganz spezielle Kommunikationssituationen, nämlich die Kommunikation von Evaluationsergebnissen bei RCTs, die Aufbereitung und Darstellung von qualitativen Daten und die Kommunikation von Akkreditierungsentscheidungen.

\section{Über welchen KANAL?}

Im Themenbereich „Kommunikationskanal“ gab es über die verschiedenen Sessions hinweg einige Diskussionen rund um das Standardformat „Evaluationsbericht“ und andere Evaluationsprodukte. Es wurde deutlich: Den „one size fits all“-Bericht gibt es nicht. Idealerweise sollten die verschiedenen Adressatinnen und Adressaten einer Evaluation auch mit verschiedenen Produkten angesprochen werden, die sich inhaltlich und in ihrer Form sicherlich voneinander unterscheiden werden. Hier müssen die Evaluierenden selbstkritisch sein und gegebenenfalls auch die Kommunikation in andere Hände (z.B. Journalismus oder Grafik-Design) abgeben, wenn die Zielgruppen der Evaluation dann besser erreicht werden können.

In diesem Zusammenhang wurde auch an mehreren Stellen betont, dass der Evaluationsprozess selbst und die Kommunikation während einer laufenden Evaluierung einen wichtigen Kanal darstellen. Gerade wenn es um die Kommunikation von negativen Ergebnissen, Fehlern und Herausforderungen geht, sollte man nicht zu lange warten. Je früher die betroffenen Stakeholder in die Analyse dieser Aspekte eingebunden werden, desto eher können sie diese Ergebnisse auch annehmen und daraus Lernprozesse ableiten. Dies gilt in besonderem Maße für die Entwicklungszusammenarbeit, wie in einer von Jelana Vajen moderierten Podiumsdiskussion mit Dorothea Auer, Gottfried von Gemmingen und Jörg Faust herausgearbeitet wurde: Obwohl die Entwicklungszusammenarbeit per se ein „Risikogeschäft“ ist, gibt es nach wie vor wenig Akzeptanz für negative Ergebnisse. Ändern können wir das wohl nur durch eine angemessene und kontextsensible Kommunikation und ganz viel Geduld.

\section{Zu WEM?}

Wie oben bereits mehrfach angeklungen ist: Wir kommunizieren im Bereich Evaluation mit einer Vielzahl ganz unterschiedlicher Gruppen: Geldgebenden, der Öffentlichkeit, Partnerorganisationen, Projektmitarbeiter(inne)n... Wichtig ist, dass diese Adressatinnen und Adressaten als „aktives Publikum“ zu verstehen sind, das Inhalte ähnlich wie bei der Rezeption von anderen Medien bedürfnisorientiert und zielgerichtet aussucht (vgl. dazu Wicke/Taddicken 2020). Diese verschiedenen Bedürfnisse und Ziele der Zielgruppen von Evaluation müssen wir im Blick halten. Dieser Aspekt wurde eigentlich in allen Sessions thematisiert. Beispielhaft seien hier zwei Sessions genannt. Zum einen diskutierten Ute Schoppmann und Susanne Frühauf in der Session des AK Schule gemeinsam mit Maria Grillitsch, Angelika Petrovic, Sylvia Opriessnig, Anja Waxenegger, Martina Hartl und Andrea 
Kulmhofer-Bommer über die Besonderheiten von Evaluationskommunikation mit Auftraggeber(inne)n aus der Politik, aber auch mit Evaluationsbeteiligten in Schule und Schulverwaltung. Zum anderen beschäftigten sich Reinhard Zweidler, Christelle Nowack und Yves Steiner in der Session des AK Umwelt damit, wie gelungene Kommunikation aussehen kann, wenn eine sozialwissenschaftlich geprägte Evaluationsperspektive auf naturwissenschaftliche Inhalte, Denk- und Vorgehensweisen beim Thema Umwelt trifft.

\section{Mit welcher WIRKUNG?}

Immer wieder wurde auf der Jahrestagung darauf hingewiesen, dass Evaluierungen ihre Wirkungen nicht erst nach Abgabe des Berichts entfalten, sondern auch während des Prozesses. Das so ermöglichte Prozesslernen (,process use“) hat Michael Q. Patton definiert als ,individual changes in thinking and behaviour and program or organizational changes in procedures and culture that occur among those involved in evaluation as a result of the learning that occurs during the evaluation process" (Patton 1997: 90). Vor allem die Kommunikation negativer Ergebnisse kann nicht früh genug während des Prozesses erfolgen. Je später Herausforderungen thematisiert werden, desto ungünstiger ist dies für die Nützlichkeit einer Evaluation.

Aber unabhängig davon, ob wir die Kommunikation während des Prozesses oder die Kommunikation der Ergebnisse ganz am Ende betrachten: Die Teilnehmenden der Jahrestagung waren sich doch ganz grundsätzlich darin einig, dass gelungene Kommunikation eine wichtige Voraussetzung dafür ist, dass Evaluation nützlich sein kann. Und das ist und bleibt ein wesentliches Anliegen der DeGEval und ihrer Mitglieder.

\section{Fazit}

Bei allem gelungenen fachlichen Austausch darüber, wie wir rund um Evaluation kommunizieren wollen, kam doch leider eine andere Form der Kommunikation bei der Online-Jahrestagung viel zu kurz: der ganz informelle, freundschaftliche Austausch zwischen uns Tagungsteilnehmerinnen und -teilnehmern. Virtuelle Kaffeepausen waren ein guter, aber natürlich nicht annähernd gleichwertiger Ersatz für die vielen Gespräche, die wir sonst zwischen einzelnen Sessions und natürlich auch im Rahmen der Abendveranstaltung haben. Dennoch möchte ich dem Organisationsteam der Jahrestagung stellvertretend für ganz viele Teilnehmende meinen großen Respekt und Dank dafür aussprechen, dass wir allen widrigen Umständen des Jahres 2020 zum Trotz an dieser inhaltlich starken und professionell durchgeführten Veranstaltung teilnehmen durften. 


\section{Literatur}

Patton, Michael Quinn (1997): Utilization-Focused Evaluation. The New Century Text. Thousand Oaks: Sage (3. Aufl.).

Taddicken, Monika/Wicke, Nina/Willems, Katharina (2020): Verständlich und kompetent? Eine Echtzeitanalyse der Wahrnehmung und Beurteilung von Expert*innen in der Wissenschaftskommunikation. In: M\&K Medien \& Kommunikationswissenschaft, 68 (1-2), S. 50-72. https://doi. org/10.5771/1615-634X-2020-1-2-50

Wicke, Nina/Taddicken, Monika (2020): Listen to the Audience(s)! Expectations and Characteristics of Expert Debate Attendants. In: Journal of Science Communication, 19 (4). Verfügbar unter: https://jcom.sissa.it/archive/19/04/JCOM_1904_2020_A02 [18.01.2021]. https://doi. org $/ 10.22323 / 2.19040202$ 